\title{
Solving the inverse problem in OCT using full-wave adjoint models
}

\author{
Callum M. Macdonald, Simon R. Arridge and Peter R.T. Munro \\ Department of Medical Physics and Biomedical Engineering, University College London, UK \\ Send correspondence to P. Munro: p.munro@ucl.ac.uk
}

\begin{abstract}
We explore the use of full-wave (Maxwell's Equations-based) forward and adjoint solvers to approach the inverse problem in Optical Coherence Tomography. We demonstrate that oscillatory artifacts in the electric susceptibility arise in the inversion process due to the intrinsic wave-nature of the forward and adjoint models. These methods however still perform well in reproducing measured data sets.

Index Terms-Optical Coherence Tomography, Microscopy, Inverse Problems
\end{abstract}

\section{INTRODUCTION}

Optical Coherence Tomography (OCT) is a threedimensional imaging technique based on low-coherence interferometry. This imaging modality is found to be readily applied in the imaging of weakly scattering materials, such as in ophthalmology where the generated images provide morphological and structural information of the human eye at resolutions up to a few micrometers. In more strongly scattering media, such as turbid tissue, image degradation due to phenomena such as multiple scattering, sample induced aberration and speckle becomes a significant issue, along with rapid reduction in signal to noise ratio as a function of imaging depth. Beyond these problems usually considered in the context of image quality, OCT does not readily produce a readout of sample-specific quantitative optical properties, such as electric susceptibility (i.e., the refractive index), of the object under observation. To find these properties, we must solve a non-trivial inverse problem.

The inverse problem of recovering the spatial distribution of the electric susceptibility requires sufficiently realistic forward models, in addition to robust inversion strategies. Previous approaches to this problem involve a variety of forward models which approximate the underlying Maxwell's Equations-based solution, such as the invocation of weak-scattering (first-order Born) approximations in the forward model [2], [3]. In this study we explore the use of full-wave solutions of Maxwell's equations for the forward and adjoint models in an attempt to faithfully represent the true nature of the scattered fields which form an OCT image. This is achieved with the employment of a Psuedo Spectral Time Domain (PSTD) solver for the incident and scattered electromagnetic fields [1].

\section{INVERSE PROBLEM}

We first investigate a more fundamental proxy to the inverse OCT problem by assuming we have access to the back-

This work was supported by the BBSRC and Royal Society scattered electric field on the upper boundary of our target medium (the boundary facing the illumination source). Here we wish to use the so-called forward-adjoint method and a full-wave PSTD model [1] of pulsed electric field propagation/scattering to find the internal electric susceptibility distribution from time-resolved or, equivalently, spectrallyresolved measurements made at this boundary. Defining a cost function, $F$, for the boundary detector, and co-located lightsource (introduced at the same boundary for simplicity), we have in terms of the $N$ discretized points on the boundary [4],

$$
F(\mathbf{p}, \vec{E})=\frac{1}{2} \sum_{n=1}^{N} \int_{0}^{T}\left(\left\|\vec{E}_{n}-\vec{E}_{n}^{m}\right\|^{2}\right) d t+g R(\mathbf{p}),
$$

where we are integrating over the time interval $[0, T], \mathbf{p}$ are our modelled parameters, $\vec{E}_{n}^{m}$ is the measured field, $\vec{E}_{n}$ is the calculated or simulated field, and $R$ is some regularization term with weighting $g$. Forming a Lagrangian for the minimization problem, it can be shown that the Lagrange multipliers themselves satisfy Maxwell's Equations, and can be computed by backpropagating the residual $\left(\vec{E}_{n}-\vec{E}_{n}^{m}\right)$ into the medium from the measurement boundary, resulting in an internal adjoint field $\vec{e}$. This means that the gradient of interest can be computed at all points within the medium using a single simulation, and with the same PSTD solver used in the forward problem, which is the main benefit of using the adjoint method. The Fréchet derivative of the augmented (i.e including constraints) cost functional $F^{a}$ with respect to the permittivity $\epsilon(\vec{r})$ is [4]

$$
\frac{\partial F^{a}}{\partial \epsilon}=-\int_{0}^{T} \vec{e} \cdot \partial t \vec{E} d t+g \epsilon_{0}^{-2} \nabla^{2} \epsilon
$$

where $\vec{e}$ and $\vec{E}$ are the internal adjoint and simulated fields respectively, and we have used first-order Tikhonov regularization. This derivative can then be used in gradientbased optimization techniques to approximate the internal susceptibility.

It is found that in this (backscattering) geometry, the counter-propagating nature of the forward and adjoint wave pulses over the interval $[0, T]$ give rise to a highly oscillitory gradient which produces artifacts in the reconstruction. These oscillations appear with a "wavelength" which is half that of the central illumination wavelength in the medium. While this gradient often will not result in an estimate of 
(a)

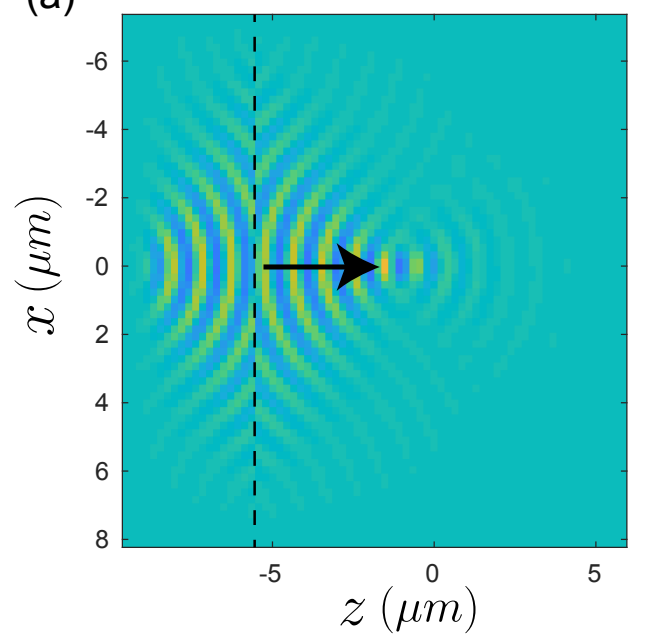

(b)

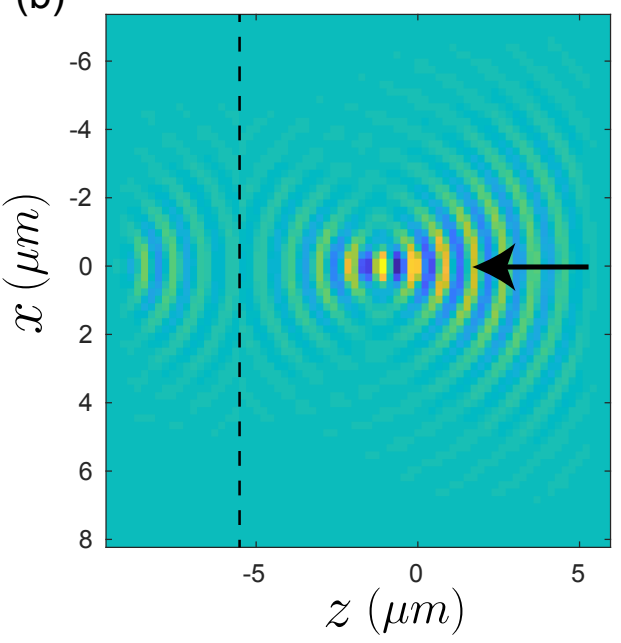

Fig. 1. (a) Back-propagated residual field introduced on dashed line, resulting in internal adjoint field, $\vec{e}$, propagating from left to right over the time interval $[T, 0]$. (b) Adjoint field propagating from right to left over the time interval $[0, T]$, opposite to the direction of propagation of the incident source field.

(a)

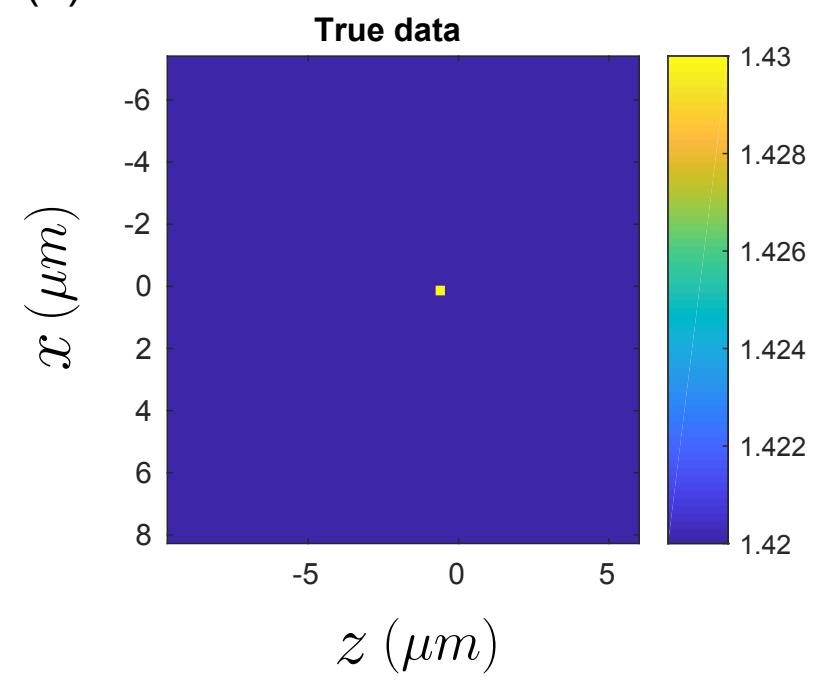

(b)

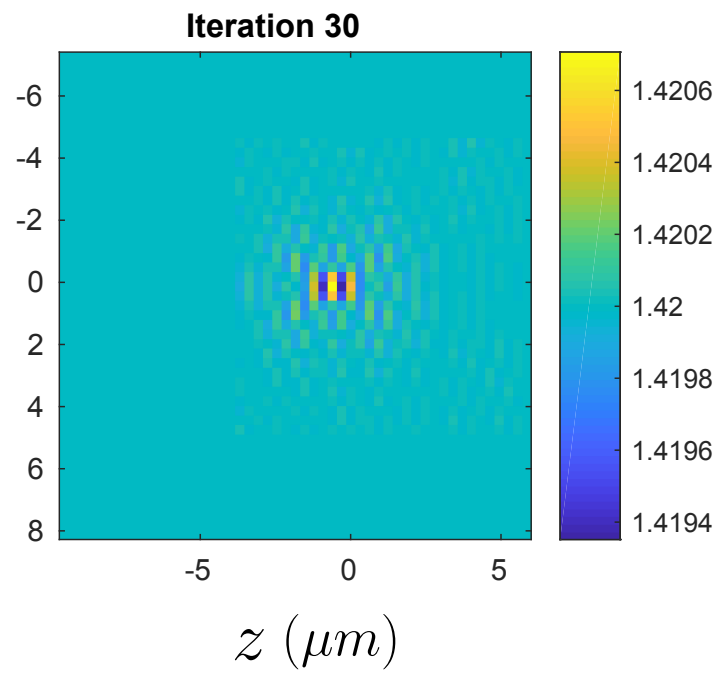

Fig. 2. (a) - Point-like refractive index inhomogeneity in a backgound medium (background refractive index $n=1.42$ ) used to produce the measured data. (b) - Reconstructed refractive index after 30 iterations. The incident light source is introduced on the left boundary travelling in the $+z$ direction in this case, with measurement of the scattered field also taking place on this boundary. Colorbar represents refractive index.

the medium which represents the ground truth susceptibiliy (refractive index) distribtuion, the measured-data is accurately replicated on the boundary. We discuss the limitations of the forward-adjoint technique using these full-wave models in the OCT geometry and potential strategies to overcome them.

\section{CONCLUSIONS}

Attempts at iterative reconstruction of sample-refractive index using OCT data have demonstrated the inverse problem to be ill-posed, principally due to non-uniqueness of the solution. This may be overcome in the future either by incorporating more prior information into the reconstruction, or acquiring more data at the time of imaging.

\section{REFERENCES}

[1] Munro, P. R. T., "Three-dimensional full wave model of image formation in optical coherence tomography," Opt. Express, 24(23), 27016-27031 (2016).

[2] Ralston, T. S., Marks, D. L., Carney, P. S., \& Boppart, S. A., "Inverse scattering for optical coherence tomography," J. Opt. Soc. Am. A, 23(5), 1027-1037 (2006).

[3] Elbau, P., Mindrinos, L., \& Scherzer, O., "Mathematical methods of optical coherence tomography," Handbook of Mathematical Methods in Imaging, 1169-1204 (2015).

[4] Rekanos, I. T., "Time-domain inverse scattering using Lagrange multipliers: An iterative FDTD-based optimization technique," J. Electromagnet. Wave., 17(2), 271-289 (2003). 